\title{
A Joint Design of Complex Signatures Sets for Multicellular Synchronous CDMA Network
}

\author{
Paul Cotae, Senior Member, IEEE
}

\begin{abstract}
A design using total squared correlation (TSC) measure for the overloaded complex spreading sequence sets on the uplink of multicellular synchronous CDMA systems is introduced. For equal power users, the design provides orthogonal Welch Bound Equality (WBE) complex spreading sequences with constant chip magnitude. We developed an algorithm, which allows obtaining a structured Gram matrix having a particular pattern for cross correlations of the multicellular signature sequence sets without alphabet constraints. By using the proposed algorithm spreading sequences with maximum equicorrelated amplitude are also obtained. The mathematical formalism is supported with selected numerical examples in the multicellular S-CDMA context.
\end{abstract}

Index Terms - Total (Weighted) Squared Correlation, generalized Welch Bound Equality (WBE) sequences, spread spectrum systems, multicellular systems, interference avoidance, and multiple access design.

\section{INTRODUCTION}

$\mathrm{T}$ HE problem of designing complex spreading sequences (a.k.a codewords or signature waveforms) for single cell synchronous code division multiple access (S-CDMA) systems is a traditional one (see [9], [12], ,13], [14], and references therein). Recently, much attention has been paid to the problem of constructing and optimizing the signature sequences for synchronous (direct sequence) DS-CDMA systems [15], and [16] in order to understand the impact of such sequences on the wireless system performances.

The current CDMA technologies for second (2G) or third generation (3G) communication systems (all based on DSCDMA, such that IS-95 A/B, cdma 2000, UTRA, W-CDMA, TD-CDMA) are suited only for low-speed continuous transmission applications such as voice, but not a good choice for high burst traffic, as in the case for future wireless communication systems (4G) [3], and [7]. Therefore a new wave of research is required for novel CDMA complex spreading design techniques.

We focus on constructing complex valued signature sets for multicellular synchronous CDMA under the total squared

Manuscript received July 12, 2006; Revised on July 15, 2007. This paper was presented in part at 2006 IEEE Radio and Wireless Symposium, RWS' 06, 17-19 Jan 2006, San Diego, and at $40^{\text {th }}$ Annual Conference on Information Sciences and Systems, CISS ‘06,22-24 March 2006, Princeton.

Paul Cotae is with the University of Texas at San Antonio, TX 78249, USA, phone 210458 6269; fax: 210458 5947; e-mail Paul.Cotae@utsa.edu . correlation (TSC) criterion, which is a measure of multiple access interferences (MAI) [25] and [26]. While the extension of some optimal results of from one cell to multiple cells is straightforward, the design of spreading sequences under TSC criterion is a more challenging task due to the amount of interference constraints that are considerably stricter than in the single-cell case ${ }^{1}$.

In a traditional deployment, base stations are located in the center of a cell. The coverage of a base station extends in both directions along the linear array. The decoding of any mobile user is undertaken by the nearest base station with co-channel interference simply treated as noise. All mobile users, which share the same two nearest base stations, will be grouped into the same cell. However, in the case of multicellular systems, we assume the cooperation of multiple base stations that share the same extended signal space and requiring that each base station to have its own power constraint.

We developed an algorithm, which allows obtaining a structured Gram matrix having a particular pattern for cross correlations of the multicellular signature sequence sets without alphabet constraints. It is an extension of the one presented in [1] in single cell case and it overcomes the limitations of the previous algorithms presented in [6], [15][17]. It is based on Gram matrix approach using TSC ([4], [5]) as a design criterion and Givens rotations [21]. By using this algorithm it is possible to obtain complex spreading sequences with maximum equicorrelated amplitude and constant chip magnitude in the absence of multipath. It is different from the algorithms presented in [8] and [24] which are based on the inverse eigenvalue problem [22], and [15]. The extension of the proposed algorithm for multipath channels [23] and unequal power users for each cell is an open problem.

The merit of this algorithm is two fold. When it is used for real numbers, then real spreading sequences are obtained, as $2 \mathrm{G}$ or $3 \mathrm{G}$ multicellular CDMA systems require them [3]. When it is used for complex numbers, then complex spreading sequences for uplink overloaded multicellular S-CDMA systems are obtained. For the particular case of the overloaded

\footnotetext{
${ }^{1}$ Some performance measure of interest, such that signal-to-interference ratio (SINR) or spectral efficiency converges to some deterministic values, which are functions of the empirical eigenvalue distribution of random matrices associated to signature spreading design. It should be noted here that the optimum spectral efficiency (without spreading) could be achieved with orthogonal spreading sequences when TSC is zero and the system load is unity. It is also known that even when the system load is higher then the unity, there exist spreading codes that incur no loss in capacity relative to multiaccess with no spreading. See also the footnote 2 .
} 
S-CDMA when all the users in a cell have the same power, this algorithm is used to obtain complex spreading sequence sets with constant chip magnitude [1], [2], and [6].

For the future wireless communication systems beyond the third generation (B3G) and 4G, where the dominant load will be high speed burst type traffic, the complex spreading sequences with constant chip magnitude for fast start-up equalization and channel estimation ([10], [11]) are definitely needed. This is also desirable for multiple antenna wireless communications [3], and equal gain unitary space-time modulation [7].

The organization of this paper is as follows. In Section II the characteristics of the uplink overloaded multicellular SCDMA systems model under the TSC criterion are described. The proposed design for constant chip magnitude is developed in Section III. The algorithm is developed in Section IV. Selected numerical results are given in Section V. Conclusions and future works are drawn in Section VI.

\section{System Model AND Total SQUAREd CoRRELATION}

Modeling the 4G CDMA network, including all the parameters such as signal propagation, fading, free space loss, etc., is a challenging task. In this paper, we focused on TSC metric, which is more tractable on the uplink than the sum capacity [16], and it was successfully applied in [25] for the next CDMA technologies in the presence of multipath. In addition, we assume that each base station has its own "policy" on power control assignment. However, for wireless cellular type systems we consider a more restrictive condition: the sum of transmitted power for each cell is constant one. In other words, the sum of elements on the main diagonal of the Gram matrix associated to each cell-spreading signature design is constant. This assumption will allow us to use a common signal space for all the cells signature design, while the powers of different signals in the multicellular system are vastly different.

We consider first the synchronous uplink vector multiple access channels (VMAC) of single-cell S-CDMA system with $K$ independent active users and the processing gain $N$ ([15], [16]). In the presence of noise vector $\boldsymbol{z}$, the received signal in one symbol interval is given by

$$
\boldsymbol{r}=\sum_{k=1}^{K} \sqrt{p_{k}} x_{k} \boldsymbol{s}_{k}+\boldsymbol{z}
$$

where for user $k, p_{k}$ is the transmitted power, $x_{k}$ is the random information symbol, and $s_{k}$ is the unitary signature sequence as $N$ dimensional column. The noise covariance matrix associated with noise vector $\boldsymbol{z}$ is $\Sigma=E\left[\boldsymbol{z}^{T}\right]$ (where by $T$ we mean transpose operation). In the case of colored noise it is assumed that the covariance matrix $\Sigma$ is positive definite with its eigenvalues $\operatorname{eig}(\Sigma)=\left[\sigma_{1}^{2}, \ldots, \sigma_{N}^{2}\right]$.

Denote by $M, K_{i}$ and $N_{i}$ the number of cells, the number of independent active users in each cell $i$ and the processing gain, respectively. Let $K_{i}>N_{i}$ as expected in the future overloaded CDMA wireless networks. Since we are dealing with deterministic signature sequences, the parameters $K_{i}$ and $N_{i}$ will be fixed. For the cell $i$ the spreading signature matrix is $S_{i}=\left[\begin{array}{llll}s_{i 1} & s_{i 2} & \ldots & s_{i K_{i}}\end{array}\right]$ of dimension $N_{i} \times K_{i}$ having the spreading sequences as columns.

The model of uplink multiple cell synchronous S-CDMA systems used in this paper is a generalization of (1) and it is given by (2) (the same model was used in [25]). Our work is different from CDMA systems with random spreading, where both the number of users and the processing gain go to infinity, while their ratio goes to same finite constant. We consider the quasi-synchronous case where chip synchronism it is assumed.

The long system received vector can be expressed as

$$
\begin{aligned}
& \boldsymbol{r}=\left[\begin{array}{l}
\boldsymbol{r}_{1} \\
\boldsymbol{r}_{2} \\
\vdots \\
\boldsymbol{r}_{M}
\end{array}\right]= \\
& =\operatorname{diag}\left(\boldsymbol{S}_{1}, \boldsymbol{S}_{2}, \ldots, \boldsymbol{S}_{M}\right)\left[\begin{array}{cccc}
\sqrt{\boldsymbol{P}_{1}} & 0 & \cdots & 0 \\
0 & \sqrt{\boldsymbol{P}_{2}} & \cdots & 0 \\
\vdots & \vdots & \ddots & \vdots \\
0 & 0 & \cdots & \sqrt{\boldsymbol{P}_{M}}
\end{array}\right]\left[\begin{array}{c}
\boldsymbol{x}_{1}^{T} \\
\boldsymbol{x}_{2}^{T} \\
\vdots \\
\boldsymbol{x}_{M}^{T}
\end{array}\right]+\left[\begin{array}{c}
\boldsymbol{z}_{1} \\
\boldsymbol{z}_{2} \\
\vdots \\
\boldsymbol{z}_{M}
\end{array}\right]
\end{aligned}
$$

where $\sqrt{\boldsymbol{P}_{i}}=\operatorname{diag}\left(\sqrt{p_{i 1}}, \ldots, \sqrt{p_{i K_{i}}}\right), \boldsymbol{x}_{i}=\left(x_{i 1}, x_{i 2}, \ldots, x_{i K_{i}}\right)$.

The composite spreading signature matrix $\boldsymbol{S}$ associated to whole overloaded multicellular system is of the form ${ }^{2}$ [25]

$$
S=S_{1} \oplus S_{2} \oplus \cdots \oplus S_{M}
$$

by considering sum of two matrices $\boldsymbol{A}$ and $\boldsymbol{B}$, i.e., $\boldsymbol{A} \oplus \boldsymbol{B}=\left(\begin{array}{cc}\boldsymbol{A} & 0 \\ 0 & \boldsymbol{B}\end{array}\right)$ [22]. In contrast with the underloaded SCDMA systems $\left(K_{i} \leq N_{i}\right)$, the composite Gram matrix of (3) is positive semidefinite (there exist at least one null

\footnotetext{
2 If we consider a composite spreading matrix of the form $\boldsymbol{S}=\left[\boldsymbol{S}_{1} \boldsymbol{S}_{2} \cdots \boldsymbol{S}_{\boldsymbol{M}}\right]$ in fact, we assume the case of equal spreading gain $N$ for each cell. This brings us back to single cell case in $N$ dimensional signal space considered in [6], [15] and [16]. When model (2) is particularized to noncolaborative scenario it allows analyzing the case where in each cell the users have different spreading gain $N_{i}$ as in the future wireless CDMA networks.
} 
eigenvalue, its determinant is zero, and Choleski decomposition with pivoting can not be applied in order to obtain the matrix $\boldsymbol{S}_{\boldsymbol{i}}$ ) may be expressed as

$$
G=S^{*} S=S_{1}^{*} S_{1} \oplus S_{2}^{*} S_{2} \oplus \cdots \oplus S_{M}^{*} S_{M}
$$

where $*$ is the complex conjugate transpose operation. The dimensionality of the extended signal space of the $\boldsymbol{G}$ matrix where the user complex spreading sequences across cells are to be designed is $\sum_{i=1}^{M} K_{i}$.

Given the composite spreading signature $\boldsymbol{S}$, by definition, the TSC metric is the Frobenius norm of the Gram matrix (4) associated to all spreading signatures in the multicellular system and it is expressed in term of its eigenvalues $\mu_{i j}$

$$
\operatorname{TSC}(\boldsymbol{S})=\|\boldsymbol{G}\|_{F}^{2}=\sum_{i=1}^{M} \sum_{j=1}^{K_{i}} \sum_{k=1}^{K_{j}}\left|\left\langle\boldsymbol{s}_{i j}, \boldsymbol{s}_{i k}\right\rangle\right|^{2}=\sum_{i=1}^{M} \sum_{j=1}^{K_{i}} \mu_{i j}^{2}
$$

TSC is minimized in [4], and [5] where for single cell case, i.e. a generic cell $i$, the corresponding spreading signature matrix $S_{i}$ satisfies the following conditions

$$
\begin{array}{ll}
\text { a) } \boldsymbol{S}_{i} \boldsymbol{S}_{i}^{*}=\frac{K_{i}}{N_{i}} \boldsymbol{I}_{N_{i}} & \text { b) } \boldsymbol{S}_{i} \boldsymbol{P}_{i} \boldsymbol{S}_{i}^{*}=\frac{\sum_{j=1}^{K_{i}} p_{i j}}{N_{i}} \boldsymbol{I}_{N_{i}}
\end{array}
$$

for unitary power users (6a) and unequal power users (6b), respectively. When the users have unitary power the condition (6a) corresponds to Welch Bound Equality sequences (WBE) [18], [19] and the condition (6b) corresponds to so called generalized WBE sequences [24], and [25] ( $\boldsymbol{I}_{N_{i}}$ is the identity matrix of order $N_{i}$ ) [21].

TSC metric defined in (5) is a measure of the total amount of interferences in the multicellular S-CDMA system and it includes as a particular case the TSC criterion considered in [4] and [5] for a single cell. In addition, it is an alternative to sum capacity formula for multicellular systems, which still is an open problem.

\section{Constant Chip Magnitude Design}

It is instructive to focus on the single cell first. We will consider the cell $i$ for convenience, after that the generalization to $M$ cells follows. In the case of equally correlated signature sequences the cross-correlation coefficient is defined as the inner product $\rho_{i}=<\boldsymbol{s}_{i j}, \boldsymbol{s}_{i k}>=\boldsymbol{s}_{i j}^{*} \boldsymbol{s}_{i k}$. For the Gram matrix $\boldsymbol{G}_{i}=\boldsymbol{S}_{i}^{*} \boldsymbol{S}_{i}$, by using (5), we have for minimum TSC

$$
\operatorname{TSC}\left(\boldsymbol{G}_{i}\right)=K_{i}+K_{i}\left(K_{i}-1\right)\left|\rho_{i}\right|^{2}=\frac{K_{i}^{2}}{N_{i}}
$$

or

$$
\left|\rho_{i}\right|^{2}=\frac{K_{i}-N_{i}}{N_{i}\left(K_{i}-1\right)}
$$

The maximum achievable crosscorrelation amplitude with $K_{i}$ equally correlated signature sequences satisfies (8). For $K_{i}=N_{i}+1$ we get $\rho_{i}= \pm 1 /\left(K_{i}-1\right)$. When $\rho_{i}=-1 /\left(K_{i}-1\right)$ the set is called a simplex [18] and the corresponding Gram matrix is

$$
\boldsymbol{G}_{i}=\left[g_{k k}=1,1 \leq k \leq K_{i}, \quad g_{k l}=-\frac{1}{K_{i}-1}, k \neq l\right]
$$

For the single cell overloaded CDMA system given in (1), where $K_{i}>N_{i}$, the Gram matrix of equally correlated sequences has the following form:

$$
\boldsymbol{G}_{i}=\left[g_{k k}=1,1 \leq k \leq K_{i}, \quad g_{k l}=\left\{\begin{array}{c} 
\pm \rho_{i} \\
\pm j \rho_{i}
\end{array}, k \neq l\right]\right.
$$

where $^{3} \quad \rho_{i}=\sqrt{\frac{K_{i}-N_{i}}{N_{i}\left(K_{i}-1\right)}}$ and $j^{2}=-1$.

Now, we extend the constant chip design for $M$ overloaded cells, $1 \leq i \leq M$, focusing on orthogonal WBE sequences for each cell. Our design for constant chip magnitude is based on the following approach. Let $e^{j \alpha_{i}}, e^{j \beta_{i}}$, and $e^{j \gamma_{i_{1}}}, \ldots, e^{j \gamma_{i_{K}-N}}$ be complex numbers with modulus one for each cell $i$, and consider the following $K_{i}$ unit vectors with $N_{i}$ components

$$
\mathbf{s}_{i 1}=\frac{1}{\sqrt{N_{i}}}\left(-e^{j \alpha_{i}}, e^{j \beta_{i}}, \ldots, e^{j \beta_{i}}\right)
$$$$
\mathbf{s}_{i 2}=\frac{1}{\sqrt{N_{i}}}\left(e^{j \beta_{i}},-e^{j \alpha_{i}}, e^{j \beta_{i}}, \ldots, e^{j \beta_{i}}\right)
$$

$\mathbf{s}_{i N_{i}}=\frac{1}{\sqrt{N_{i}}}\left(e^{j \beta_{i}}, e^{j \beta_{i}}, \ldots, e^{j \beta_{i}},-e^{j \alpha_{i}}\right)$,

$$
\mathbf{s}_{i\left(N_{i}+1\right)}=\frac{1}{\sqrt{N_{i}}}\left(e^{j \gamma_{i 1}}, e^{j \gamma_{i 1}}, \ldots, e^{j \gamma_{i 1}}\right)
$$

\footnotetext{
${ }^{3}$ For real minimum WBE sequences (MWBE) $g_{i j}= \pm \rho, i \neq j$ and for complex MWBE sequences $g_{i j}= \pm j \rho, i \neq j[18]$.
} 
$\mathbf{s}_{i K_{i}}=\frac{1}{\sqrt{N_{i}}}\left(e^{j \gamma_{i_{K-N}}}, \ldots, e^{j \gamma_{i K-N}}\right)$

In order to construct the Gram matrix given in (10) using the pattern imposed in (11), the following system of equations in unknown $\alpha_{i}, \beta_{i}, \gamma_{i}$ must be solved

$$
\begin{gathered}
\left|<\mathbf{s}_{i k}, \mathbf{s}_{i l}>\right|=\frac{1}{N_{i}}\left|-e^{j\left(\alpha_{i}-\beta_{i}\right)}-e^{-j\left(\alpha_{i}-\beta_{i}\right)}+N_{i}-2\right|=\rho_{i}, \\
1 \leq i \leq M, \quad 1 \leq k, l \leq N_{i} \\
\left|<\mathbf{s}_{i k}, \mathbf{s}_{i l}>\right|=\frac{1}{N_{i}}\left|-e^{j\left(\alpha_{i}-\gamma_{i l}\right)}+\left(N_{i}-1\right) e^{j\left(\beta_{i}-\gamma_{i l}\right)}\right|=\rho_{i}, \\
1 \leq i \leq M, \quad, 1 \leq k \leq N_{i}, \quad 1 \leq l \leq K_{i}-N_{i}-1 \\
<\mathbf{s}_{i k}, \mathbf{s}_{i K_{i}}>=\frac{1}{N}\left[-e^{j\left(\alpha_{i}-\gamma_{K-N}\right)}+(N-1) e^{j\left(\beta_{i}-\gamma_{K-N}\right)}\right] \\
=\left\{\begin{array}{l}
-\rho_{i} \\
j \rho_{i}
\end{array}, 1 \leq \mathrm{i} \leq \mathrm{M}, 1 \leq k \leq K_{i}-1\right.
\end{gathered}
$$

The equation (12a) is a quadratic one whose discriminator depends on the values of $K_{i}$ and $N_{i}$ being associated to the first $N_{i}$ users. The equation (12b) corresponds to the next $K_{i}-N_{i}-1$ overloaded users, and the last equation considers the inner product of the first $\left(K_{i}-1\right)$ users with the last one. This imposed particular form is discussed in Lemma 4.1 (the next Section). By solving the system (12a)-(12c) using standard numerical methods [21] we obtained for a single cell with the signal dimension $N \in[1,6]$ the following results:

TABLE I

COMPLEX SPREADING SEQUENCES WITH REAL MAXIMUM EQUAL CROSSCORRELATION AMPLITUDE ${ }^{4}$

\begin{tabular}{||l|l|l|l|l|l|l|l||}
\hline \hline $\mathrm{K}$ & $3^{+}$ & $4^{+}$ & $5^{+}$ & 6 & $7^{+}$ & 10 & 16 \\
$\mathrm{~N}$ & 2 & 3 & 4 & 3 & 6 & 5 & 6 \\
\hline
\end{tabular}

TABLE II

COMPLEX SPREADING SEQUENCES WITH COMPLEX MAXIMUM EQUAL CROSSCORRELATION COEFFICIENT ${ }^{5}$

\begin{tabular}{|c|c|c|c|c|c|c|c|c|c|}
\hline $\mathrm{K}$ & 4 & 7 & $7^{++}$ & 9 & $11^{+}$ & $11^{++}$ & 12 & $13^{++}$ & 1 \\
\hline $\mathrm{N}$ & 2 & 3 & 4 & 3 & 5 & 6 & 6 & 4 & 4 \\
\hline
\end{tabular}

The Jacobi method [21] of calculating the eigenvalues of a

${ }^{4}$ The + corresponds to the simplex case (K, K-1) and one of the nice feature of the proposed algorithm in the next section is that generates complex spreading sequences with constant chip magnitude.

${ }^{5}$ The ++ cases were reported in [6]. symmetric matrix use Givens (Jacobi) rotations. The idea behind Jacobi's method is to reduce systematically the off diagonal entries of a matrix. Under TSC design we are going in the opposite direction. The proposed design for constant chip magnitude overcomes the limitation of numerical search method based on generalized Lloyd algorithm developed in [6] due to highly structured matrix ((7) in [6]) which does not satisfy the bandwidth constraint for each cell as it is discussed next.

\section{THE PROPOSED ALGORITHM}

If we know the optimal eigenvalues of Gram matrix specified by (5), then based on (4), we need to construct a structured Gram matrix of the form required by (4), and at the same time, we need to preserve the bandwidth ${ }^{6}$ of spreading sequences of each cell. The algorithm proposed in [15], (reproduced completely with its original proof in [17]) and those used in [16] based on the so called T-transform [20], may fail to generate valid complex spreading sequences for overloaded multicellular S-CDMA systems under TSC measure defined in [5].

The main drawbacks of the algorithms presented in [15][17] (when they are used for construction of the complex spreading sequences for overloaded multicellular CDMA systems) is the requirement of ordering the eigenvalues of composite Gram matrix [8].

Our algorithm generalizes the algorithms given in [1]; it is amenable to online implementation due to the finite number of steps required for convergence. Focusing on TSC, the proposed algorithm might be used for decentralized transmitter adaptation in multicellular systems and it does not require updates of sequences at each step. Hence it is suitable for a distributed implementation. The algorithm is based on the following theorem [1] that allows constructing a structured orthogonal matrix necessary in generating matrices in (4).

Theorem 4.1: Given the vectors $\boldsymbol{x}=\left(\boldsymbol{x}_{1}, \boldsymbol{x}_{2}, \ldots, \boldsymbol{x}_{M}\right)$ and $\boldsymbol{y}=\left(\boldsymbol{y}_{1}, \boldsymbol{y}_{2}, \ldots, \boldsymbol{y}_{M}\right)$ such that each vector $\boldsymbol{x}_{i}$ is majorized by the vector $\boldsymbol{y}_{i}$ (of the same length $N_{i}$ ) then there exist an orthogonal matrix $\boldsymbol{U}$ such that the diagonal entries of the matrix $\boldsymbol{U}^{T} \operatorname{diag}(\boldsymbol{y}) \boldsymbol{U}$ are the components of $\boldsymbol{x}$ and $\boldsymbol{U}$ can be written as the product of at most $M(N-1)$ orthogonal rotations.

The proof is given in the Appendix ${ }^{7}$.

\footnotetext{
${ }^{6}$ The bandwidth is specified by the dimensionality of initial signal space of the dimension $N_{i}$.

${ }^{7}$ This theorem is a generalization of those presented in [27] and [28], which is referring to a single majorization relation between two vectors of the same length. The extension of complex spreading sequence design to multiple cells context is not trivial due to different vector lengths in each cell.
} 


\section{Algorithm}

Input: $K_{i}, N_{i}, \boldsymbol{x}_{i}=\boldsymbol{1}_{K_{i}}, \boldsymbol{y}=\left(K_{i} / N_{i}, \ldots, K_{i} / N_{i}, 0, \ldots, 0\right)$.

Output: The Gram matrix $\boldsymbol{G}$ such that $\operatorname{diag}(\boldsymbol{G})=\boldsymbol{x}$, $e i g(\boldsymbol{G})=\boldsymbol{y}$ and $\rho_{i}$ are given by (8).

\section{Update:}

1. For each cell $j, \quad 1 \leq j \leq M$ :

2. Initialization $i=1, g_{11}=x_{[1]}$. Construct the matrix $\mathbf{G}_{0}=\operatorname{diag}(\mathbf{y})$. Construct the matrix $\mathbf{V}_{1}$ given by (13) for $p=1$ and $q=K_{j}$. Let $\mathbf{U}_{1}=\mathbf{V}_{1}$. Construct the matrix $\mathbf{G}_{1}=\mathbf{U}_{1}^{T} \mathbf{G}_{0} \mathbf{U}_{1}$.

3. $i=i+1$

4. Construct the matrix $\mathbf{V}_{i}$ for $p=i$ and $q=K_{j}$. Let $\mathbf{U}_{i}=\mathbf{V}_{i}$. Construct $\mathbf{G}_{i+1}=\mathbf{U}_{i}^{T} \mathbf{G}_{i} \mathbf{U}_{i}$ and $\mathbf{U}=\mathbf{V}_{1} \mathbf{V}_{2} \ldots \mathbf{V}_{i}$.

5. For $K_{j}=N_{j}+1$, solve the system given in (9) for $<\mathbf{s}_{i}, \mathbf{s}_{j}>=-\frac{1}{K_{j}-1}, 1 \leq i \leq K_{j}-1$ for obtaining $\alpha_{j}$ and $\beta_{j}$ for an imposed $\gamma_{j}$.

6. For $K_{j}>N_{j}+1$, solve (9) for complex spreading sequences.

7. Termination if $i=K_{j}-1$.

8. Termination if $j=M-1$.

The general form of the orthogonal matrix $\boldsymbol{V}$ used for $(p, p)$ and $(q, q)$ elements on main diagonal of Gram matrix is

$\boldsymbol{V}=\left[\begin{array}{lllllllllll}1 & \cdots & 0 & 0 & \cdots & & & \cdots & 0 & \cdots & 0 \\ \vdots & \ddots & \vdots & \vdots & & & & & \vdots & & \vdots \\ 0 & \cdots & 1 & 0 & \cdots & & & \cdots & 0 & \cdots & 0 \\ 0 & \cdots & 0 & \cos \varphi & 0 & \cdots & 0 & \sin \varphi & 0 & \cdots & 0 \\ \vdots & & \vdots & 0 & 1 & \cdots & 0 & 0 & \vdots & & \vdots \\ & & & \vdots & 0 & \ddots & \vdots & \vdots & & & \\ \vdots & & \vdots & 0 & \vdots & \ddots & 1 & 0 & \vdots & & \vdots \\ 0 & \cdots & 0 & -\sin \varphi & 0 & \cdots & 0 & \cos \varphi & 0 & \cdots & 0 \\ & & & 0 & \cdots & & \cdots & 0 & 1 & \cdots & 0 \\ \vdots & & \vdots & \vdots & & & & & \vdots & \ddots & \vdots \\ 0 & \cdots & 0 & 0 & \cdots & & \cdots & 0 & 0 & \cdots & 1\end{array}\right] q$

Lemma 4.1: The Gram matrix obtained with the proposed Algorithm has one of the following patterns:
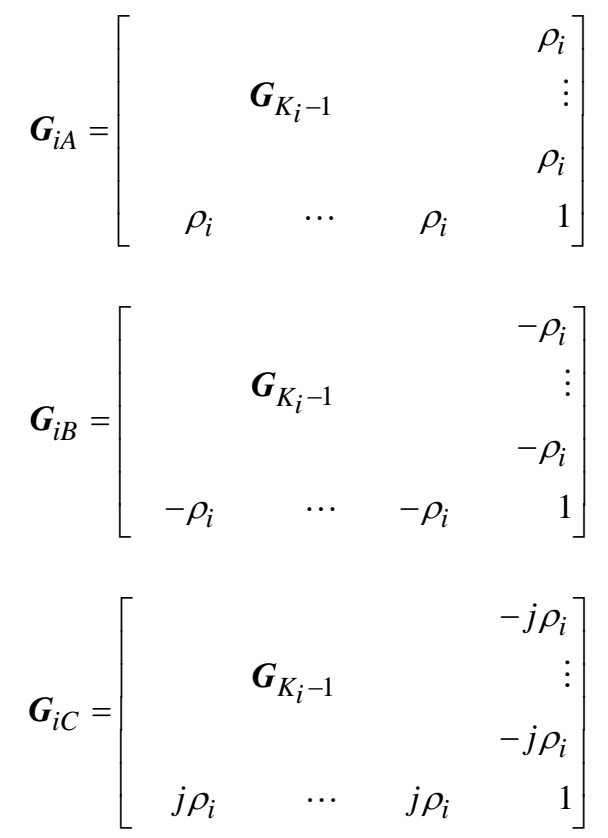

Proof: The Algorithm starts with $p=1$ and $q=K_{j}$ imposing for the element $g_{11}=K_{j} / N_{j}$ of $\boldsymbol{G}_{0}$ the value 1 . After the first iteration the main diagonal of $\boldsymbol{G}_{1}$ becomes $\operatorname{diag}\left(\boldsymbol{G}_{1}\right)=(1, \underbrace{K_{j} / N_{j}, \ldots, K_{j} / N_{j}}_{N_{j}-1}, \underbrace{0, \ldots, 0}_{K_{j}-N_{j}},\left(K_{j}-N_{j}\right) / N_{j})$

since the $\operatorname{trace}\left(\boldsymbol{G}_{0}\right)=K_{j}$.

The Gram matrix obtained with the algorithm after the first iteration can be written as

$$
\boldsymbol{G}_{1}=\left[\begin{array}{cc}
1 & \boldsymbol{x} \\
\boldsymbol{x}^{T} & \boldsymbol{G}_{11}
\end{array}\right]
$$

where $\boldsymbol{x}$ is of order $1 \times\left(K_{j}-1\right)$ and $\boldsymbol{G}_{11}$ is a matrix of order $\left(K_{j}-1\right) \times\left(K_{j}-1\right)$ with the eigenvalues

$$
\operatorname{eig}\left(\boldsymbol{G}_{11}\right)=(\underbrace{K_{j} / N_{j}, \ldots, K_{j} / N_{j}}_{N_{j}-1}, \underbrace{0, \ldots, 0}_{K_{j}-N_{j}}\left(K_{j}-N_{j}\right) / N_{j}) \text {. }
$$

The Algorithm will continue with $p=2$ and $q=K_{j}$. We can apply this procedure at most $K_{j}-1$ times. Since the Frobenius norm is preserved by orthogonal transformations, we find that, after the first iteration, the TSC is given by

$$
\begin{aligned}
\operatorname{TSC}\left(\boldsymbol{G}_{0}\right) & =\frac{K_{j}^{2}}{N_{j}}=1+2|<\boldsymbol{x}, \boldsymbol{x}>|^{2}+\operatorname{TSC}\left(\boldsymbol{G}_{11}\right) \\
& =1+2|<\boldsymbol{x}, \boldsymbol{x}>|^{2}+\frac{K_{j}^{2}-2 K_{j}+N_{j}}{N_{j}}
\end{aligned}
$$


Thus, under the TSC design, the absolute value of the inner product for the vector $\boldsymbol{x}$ is exactly given by (10). In the last iteration we obtain $\boldsymbol{G}=\left[\begin{array}{cc}\boldsymbol{G}_{K_{i}-1} & \boldsymbol{y} \\ \boldsymbol{y}^{T} & 1\end{array}\right]$. Again, since TSC is preserved by using Givens rotations, we have

$$
\begin{aligned}
\operatorname{TSC}\left(\boldsymbol{G}_{0}\right) & =\operatorname{TSC}(\boldsymbol{G})=1+2|<\boldsymbol{y}, \boldsymbol{y}>|^{2}+\operatorname{TSC}\left(\boldsymbol{G}_{K_{i}-1}\right) \\
& =1+2|<\boldsymbol{y}, \boldsymbol{y}>|^{2}+\operatorname{TSC}\left(\boldsymbol{G}_{11}\right)
\end{aligned}
$$

We observe that, due to symmetry, the column vector $\boldsymbol{y}$ has all equal elements. Thus, the vector $\boldsymbol{y}$ can have the form $\boldsymbol{y}^{T}=\left(\rho_{i}, \ldots, \rho_{i}\right) \quad$ or $\quad \boldsymbol{y}^{T}=\left(-\rho_{i}, \ldots,-\rho_{i}\right) \quad$ for $\quad$ real crosscorrelation case and $\boldsymbol{y}^{T}=\left(j \rho_{i}, \ldots, j \rho_{i}\right)$ or $\boldsymbol{y}^{T}=(-j \rho, \ldots,-j \rho)$ for complex crosscorrelation case, which concludes the lemma. Since the Gram matrix is normal ( $\boldsymbol{G}^{*}=\boldsymbol{G} \boldsymbol{U}$ for some unitary matrix $\boldsymbol{U}$ ) we will prove next, that for each pattern in (14), there exist a unique orthogonal matrix $\mathbf{V}$, which considerably simplifies the design.

Lemma 4.2: The orthogonal matrix $\mathbf{V}$ used to construct matrices given by (14) is unique.

Proof: The Gram matrix of order $K_{i}$ given by (14) is a normal matrix since it commutes with its Hermitian adjoint. By Theorem 2.5.8 [22] there is an orthogonal matrix $\mathbf{Q}$ such that

$$
\boldsymbol{Q}^{T} \boldsymbol{G} \boldsymbol{Q}=\operatorname{diag}\left(\boldsymbol{G}_{1}, \boldsymbol{G}_{2}, \ldots, \boldsymbol{G}_{K_{i}}\right), \quad 1 \leq k \leq K_{i}
$$

where each $\mathbf{G}_{j}$ is either a real 1 by 1 matrix or is a real 2 by 2 matrix of the following form

$$
\boldsymbol{G}_{j}=\left[\begin{array}{cc}
\alpha_{j} & \beta_{j} \\
-\beta_{j} & \alpha_{j}
\end{array}\right]
$$

With the proposed Algorithm we can find an orthogonal matrix $\mathbf{U}$ such that

$$
\widetilde{\boldsymbol{G}}_{1}=\boldsymbol{U}^{T} \operatorname{diag}(\underbrace{K_{i} / N_{i}, \ldots, K_{i} / N_{i}}_{N_{i}}, \underbrace{0, \ldots, 0}_{K_{i}-N_{i}}) \boldsymbol{U}
$$

The value of the determinant of $\mathbf{V}$ given by (13) is 1 so $\mathbf{U}$ is a unitary matrix. From (16) there is an orthogonal matrix $\mathbf{Q}$ such that

$$
\widetilde{\boldsymbol{G}}_{2}=\boldsymbol{Q}^{T} \operatorname{diag}(\underbrace{K_{i} / N_{i}, \ldots, K_{i} / N_{i}}_{N_{i}}, \underbrace{0, \ldots, 0}_{K_{i}-N_{i}}) \boldsymbol{Q}
$$

It is easy to check that $\widetilde{\mathbf{G}}_{1}$ and $\widetilde{\mathbf{G}}_{2}$ are real commuting normal matrices and by Theorem 2.5.15 [22] there exists a unique real orthogonal matrix $\mathbf{P}$ such that $\mathbf{P}^{T} \widetilde{\mathbf{G}}_{1} \mathbf{P}$ and $\mathbf{P}^{T} \widetilde{\mathbf{G}}_{2} \mathbf{P}$ to be of the form (13) which concludes the proof.

\section{NUMERICAL RESUlTS}

Experiment 5.1 Generating orthogonal WBE complex spreading sequences with constant chip magnitude.

Consider two cells with having 8 users with the same spreading gain $K_{1}=4, N_{1}=3$ and $K_{2}=4, N_{2}=3$. The following vector $\boldsymbol{y}=(4 / 3,4 / 3,4 / 3,0,4 / 3,4 / 3,4 / 3,0)$ gives the distribution of the optimal eigenvalues used for construction. Running the proposed algorithm with the above data we obtained the following matrices

$$
\boldsymbol{G}=\left[\begin{array}{rr}
\boldsymbol{G}_{1} & 0 \\
0 & \boldsymbol{G}_{2}
\end{array}\right] \quad \boldsymbol{S}=\left[\begin{array}{rr}
\boldsymbol{S}_{1} & 0 \\
0 & \boldsymbol{S}_{2}
\end{array}\right]
$$

where the Gram matrices for the users are obtained as

$$
\begin{aligned}
& \boldsymbol{G}_{1}=\left[\begin{array}{rrrr}
1.0000 & -0.3333 & -0.3333 & 0.3333 \\
-0.3333 & 1.0000 & -0.3333 & 0.3333 \\
-0.3333 & -0.3333 & 1.0000 & 0.3333 \\
0.3333 & 0.3333 & 0.3333 & 1.0000
\end{array}\right] \\
& \boldsymbol{G}_{2}=\left[\begin{array}{rrrr}
1.0000 & -0.3333 & -0.3333 & -0.3333 \\
-0.3333 & 1.0000 & -0.3333 & -0.3333 \\
-0.3333 & -0.3333 & 1.0000 & -0.3333 \\
-0.3333 & -0.3333 & -0.3333 & 1.0000
\end{array}\right]
\end{aligned}
$$

and the corresponding complex spreading sequences with constant chip magnitude are obtained according to Lemma 4.1:

$\boldsymbol{S}_{1}=\left[\begin{array}{rrrr}0.5556+0.1571 j & 0.5556+0.1571 j & -0.5556-0.1571 j & 0.5556+0.1571 j \\ 0.5556+0.1571 j & -0.5556-0.1571 j & -0.5556-0.1571 j & -0.5556-0.1571 j \\ 0.5556+0.1571 j & -0.5556-0.1571 j & 0.5556+0.1571 j & 0.5556+0.1571 j\end{array}\right]$ $\boldsymbol{S}_{2}=\left[\begin{array}{rrrr}0.3333+0.4714 j & 0.3333+0.4714 j & -0.3333-0.4714 j & 0.3333-0.4714 j \\ 0.3333-0.4714 j & -0.3333+0.4714 j & -0.3333+0.4714 j & 0.3333-0.4714 j \\ 0.3333+0.4714 j & -0.3333-0.4714 j & 0.3333+0.4714 j & -0.3333-0.4714 j\end{array}\right]$

One might think it is possible to design complex spreading sequences for individual cells and concatenate their correlation matrices as diagonal blocks in a composite blockdiagonal correlation matrix as long as the cells operate orthogonally to each other. This is true in a non-collaborative scenario when users communicate independently and are 
supervised by only one base station ${ }^{8}$.

In this case the $T$-transform provides the same results as the algorithm proposed in this paper. However, in a collaborative scenario and in the same extended signal space, users need to have the same signature waveforms when they are "seen" by different base stations. This condition is satisfied if there exist a matrix $\boldsymbol{U}$ as it is provided by Theorem 4.1.

Experiment 5.2 Generating orthogonal WBE complex spreading sequences with real maximum equal crosscorrelation amplitude:

We also consider two cells with 12 users with the same spreading gain $K_{1}=6, N_{1}=3$ and $K_{2}=6, N_{2}=3$. The following vectors $\quad \boldsymbol{x}=(\underbrace{1,1, \ldots, 1}_{12})$ and $\boldsymbol{y}=(2,2,2,0,0,0,2,2,2,0,0,0)$ give the distribution of the diagonal of composite Gram matrix and the optimal eigenvalues used for construction. Running the proposed algorithm with the above data we obtained the following structured Gram matrix

$$
\boldsymbol{G}=\left[\begin{array}{rr}
\boldsymbol{G}_{1} & 0 \\
0 & \boldsymbol{G}_{2}
\end{array}\right]
$$

where the Gram matrices for the users are obtained again with patterns specified by Lemma 4.1:

$$
\begin{aligned}
& \boldsymbol{G}_{1}=\frac{\sqrt{5}}{5}\left[\begin{array}{rrrrrr}
\sqrt{5} & 1 & -1 & 1 & -1 & -1 \\
1 & \sqrt{5} & -1 & -1 & 1 & -1 \\
-1 & -1 & \sqrt{5} & 1 & 1 & -1 \\
1 & -1 & 1 & \sqrt{5} & -1 & -1 \\
-1 & 1 & 1 & -1 & \sqrt{5} & -1 \\
-1 & -1 & -1 & -1 & -1 & \sqrt{5}
\end{array}\right] \\
& \boldsymbol{G}_{2}=\frac{\sqrt{5}}{5}\left[\begin{array}{rrrrrr}
\sqrt{5} & 1 & -1 & 1 & -1 & 1 \\
1 & \sqrt{5} & -1 & -1 & 1 & 1 \\
-1 & -1 & \sqrt{5} & 1 & 1 & 1 \\
1 & -1 & 1 & \sqrt{5} & -1 & 1 \\
-1 & 1 & 1 & -1 & \sqrt{5} & 1 \\
1 & 1 & 1 & 1 & 1 & \sqrt{5}
\end{array}\right]
\end{aligned}
$$

As in the previous experiment we can observe that the bandwidth of spreading sequences in each cell, specified by $N_{1}$ and $N_{2}$, respectively, is preserved in the signal space of

\footnotetext{
${ }^{8}$ The notion of collaborative base stations is crucial to the idea of intercell interference mitigation. The extension of our results to overloaded "cognitive radios" is left for future research. Thank you to anonymous reviewer for this perspective.
}

dimension $K_{1}+K_{2}$ of the composite Gram matrix .

Experiment 5.3 Generating orthogonal complex spreading sequences with complex maximum equal crosscorrelation amplitude:

For this experiment we used the optimal eigenvalue vector $\boldsymbol{y}=(2,2,0,0,2,2,0,0)$ and the corresponding Gram matrices for each cell with four users are obtained as

$$
\begin{aligned}
& \boldsymbol{G}_{1}=\left[\begin{array}{cccc}
1 & \frac{-j}{\sqrt{3}} & \frac{-j}{\sqrt{3}} & \frac{j}{\sqrt{3}} \\
\frac{j}{\sqrt{3}} & 1 & \frac{j}{\sqrt{3}} & \frac{j}{\sqrt{3}} \\
\frac{j}{\sqrt{3}} & \frac{-j}{\sqrt{3}} & 1 & \frac{j}{\sqrt{3}} \\
\frac{-j}{\sqrt{3}} & \frac{-j}{\sqrt{3}} & \frac{-j}{\sqrt{3}} & 1
\end{array}\right] \\
& \boldsymbol{G}_{2}=\left[\begin{array}{cccc}
1 & \frac{-j}{\sqrt{3}} & \frac{-j}{\sqrt{3}} & \frac{-j}{\sqrt{3}} \\
\frac{j}{\sqrt{3}} & 1 & \frac{j}{\sqrt{3}} & \frac{-j}{\sqrt{3}} \\
\frac{j}{\sqrt{3}} & \frac{-j}{\sqrt{3}} & 1 & \frac{-j}{\sqrt{3}} \\
\frac{j}{\sqrt{3}} & \frac{j}{\sqrt{3}} & \frac{j}{\sqrt{3}} & 1
\end{array}\right]
\end{aligned}
$$

The above two matrices specified by Lemma 4.1 are in fact the same since we can check that $\boldsymbol{G}_{1}^{*}=\boldsymbol{G}_{2}$.

\section{CONCLUSIONS AND FUTURE WORK}

We developed a distributed algorithm for 4G wireless communications providing complex spreading sequences with constant chip magnitude and maximum cross correlation amplitude for multicellular S-CDMA. In this paper we have focused on symbol-synchronous CDMA systems in the presence of AWGN. The extension of our results to the asynchronous situation and considering colored noise is interesting and also an important open problem. Our current efforts are directed towards solving this important open question.

\section{APPENDIX}

Proof of the Theorem 4.1:

a) For the particular case when $M=1$ the Theorem 4.1 is the same with Theorem 4.1 [2]. 
b) Consider $M>1$ and we use induction on $N$. Without loss of generality we can assume within a cell $i$ the eigenvalues $y_{i}$ (of $\boldsymbol{y}_{i}$ ) and diagonal elements $x_{i}$ (of $\boldsymbol{x}_{\boldsymbol{i}}$ ) are arranged in decreased order $y_{1} \geq y_{2} \geq \ldots \geq y_{N}$ and $x_{1} \geq x_{2} \geq \ldots \geq x_{N}$. The vector $\boldsymbol{x}$ is said to be majorized by the vector $\boldsymbol{y}$, denoted $\boldsymbol{x} \prec \boldsymbol{y}$ if [20]

$$
\begin{aligned}
& \text { a) } \sum_{i=1}^{k} x_{i} \leq \sum_{i=1}^{k} y_{i}, \quad k=1,2, \ldots, N-1 \\
& \text { b) } \sum_{i=1}^{N} x_{i}=\sum_{i=1}^{N} y_{i}
\end{aligned}
$$

i) Verify for $M=2$ and $N=2$. We start with $M=1$. Condition (22) is equivalent to $x_{1} \leq y_{1}, x_{1}+x_{2}=y_{1}+y_{2}$ and becomes $\quad y_{1} \geq x_{1} \geq x_{2} \geq y_{2} \quad$ since $x_{1}=y_{1}+y_{2}-x_{2} \geq y_{1}+y_{2}-x_{1} \geq y_{2}$ and $x_{2}=y_{1}+y_{2}-x_{1}$. If $y_{1}=y_{2}$ the proof is trivial. Otherwise $y_{1}>y_{2}$ and it follows from (22) that $y_{1} \geq x_{1} \geq x_{2}>y_{2}$. We can use Lemma 4.1 [2] imposing for the elements of matrix $\boldsymbol{A}$ the values $x=x_{1}, z=x_{2}$. After solving for $\left(x_{2}-y_{1}\right) \tan ^{2}(\varphi)-2 y \tan (\varphi)+\left(x_{1}-y_{1}\right)=0$ we can find the orthogonal matrix $\boldsymbol{V}$ of order 2 in one step. Hence the theorem holds for $M=1$ and $N=2$ as we expected.

For the matrix $\operatorname{diag}\left(\mathbf{y}_{1}, \mathbf{y}_{2}\right)$ there exist a rotation matrix $\boldsymbol{V}$ of order 4 such that the $(1,1),(2,2),(3,3)$ and $(4,4)$ elements of $\boldsymbol{V}^{T} \operatorname{diag}\left(\boldsymbol{y}_{1}, \boldsymbol{y}_{2}\right) \boldsymbol{V}$ are $x_{1}, x_{2}=y_{1}+y_{2}-x_{1}, x_{3}$, $x_{4}=y_{3}+y_{4}-x_{3}$, respectively [2]. We need to apply Lemma 4.1 [2] twice to solve for eigenvalues $\mathbf{y}_{1}, \mathbf{y}_{2}$. Hence the theorem holds for $M=2, N=2$ and 2(2-1) orthogonal rotations are necessary.

ii) Now suppose that theorem holds for $M=2$ cells, $N \geq 2$ and we will prove for $N+1$. The main idea is to use two block orthogonal rotation matrices of order $2(N-1)$ such that

$$
\begin{aligned}
& \boldsymbol{U}_{1}=\operatorname{diag}\left(\boldsymbol{I}_{N-1}, \boldsymbol{V}_{1}, \boldsymbol{I}_{N-1}, \boldsymbol{V}_{2}\right) \\
& \boldsymbol{U}_{2}=\operatorname{diag}\left(\boldsymbol{U}_{N}, 1, \boldsymbol{U}_{N}, 1\right)
\end{aligned}
$$

where $\boldsymbol{V}_{1}$ and $\boldsymbol{V}_{2}$ are obtained as in [2].

Regarding the cellular CDMA context where we assumed the same processing gain $N$ in all cells, it is interesting to note that from this point on in proving the Theorem 4.1 we can also assume different processing gain in different cells $i$ and $j$. However, for sake of simplicity we will continue with the vectors of the same lengths. Let $\boldsymbol{\Lambda}=\operatorname{diag}\left(y_{i 1}, y_{i 2}, \ldots, y_{i N}, y_{i N+1}, y_{j 1}, y_{j 2}, \ldots, y_{j N}, y_{j N+1}\right)$ be a diagonal matrix of order $2(N+1)$. By Schur's theorem [20] there exist a Hermitian matrix with the eigenvalues $y_{i 1}, y_{i 2}, \ldots, y_{i N}, y_{i N+1}, y_{j 1}, y_{j 2}, \ldots, y_{j N}, y_{j N+1}$ and diagonal elements $x_{i 1}, x_{i 2}, \ldots, x_{i N}, x_{i N+1}, x_{j 1}, x_{j 2}, \ldots, x_{j N}, x_{j N+1}$

satisfying (22). We can find for each cell the smallest integers $m$ and $n$ such that $y_{i m} \geq x_{i N+1} \geq y_{i m+1}$ and $y_{j n} \geq x_{j N+1} \geq y_{j n+1}$, respectively. Considering the eigenvalues $y_{i m}$ and $y_{j n}$, we can permute the diagonal elements of $\boldsymbol{\Lambda}$ to obtain the matrix

$$
\begin{gathered}
\Lambda_{i j}=\operatorname{diag}\left(y_{i 1}, y_{i 2}, \ldots, y_{i m-1}, y_{i m+1}, \ldots, y_{i N}, y_{i m}, y_{i N+1},\right. \\
\left.y_{j 1}, y_{j 2}, \ldots, y_{j n-1}, y_{j n+1}, \ldots, y_{j N}, y_{j n}, y_{j N+1}\right)
\end{gathered}
$$

Using the matrix $\boldsymbol{U}_{1}$ we obtain

$$
\operatorname{diag}\left(\boldsymbol{U}_{1}^{T} \boldsymbol{\Lambda}_{i j} \boldsymbol{U}_{1}\right)=\operatorname{diag}\left(\boldsymbol{\Lambda}_{1}, x_{i N+1}, \boldsymbol{\Lambda}_{2}, x_{j N+1}\right)
$$

where

$$
\boldsymbol{\Lambda}_{1}=\operatorname{diag}\left(y_{i 1}, y_{i 2}, \ldots, y_{i m-1}, y_{i m+1}, \ldots, y_{i N}, y_{i m}+y_{i N+1}-x_{i N+1}\right)
$$

and

$$
\begin{gathered}
\boldsymbol{\Lambda}_{2}=\operatorname{diag}\left(y_{j 1}, y_{j 2}, \ldots, y_{j n-1}, y_{j n+1}, \ldots,\right. \\
\left.y_{j N}, y_{j n}+y_{j N+1}-x_{j N+1}\right)
\end{gathered}
$$

Let $\mu_{1}$ be the following eigenvalue vector $\boldsymbol{\mu}_{1}=\left(y_{i 1}, y_{i 2}, \ldots, y_{i m-1}, y_{i m+1}, \ldots, y_{i N}, y_{i m}+y_{i N+1}-x_{i N+1}\right)$ and $\boldsymbol{\mu}_{2}=\left(y_{j 1}, y_{j 2}, \ldots, y_{j n-1}, y_{j n+1}, \ldots, y_{j N}, y_{j n}+y_{j N+1}-x_{j N+1}\right)$, respectively. Consider the initial elements on diagonal i.e. the vectors $\boldsymbol{\eta}_{1}=\left(x_{i 1}, x_{i 2}, \ldots, x_{i N}\right)$ and $\boldsymbol{\eta}_{2}=\left(x_{j 1}, x_{j 2}, \ldots, x_{j N}\right)$, respectively. Now, what remains is to prove that $\left(\boldsymbol{\eta}_{1}, \boldsymbol{\eta}_{2}\right) \prec\left(\boldsymbol{\mu}_{1}, \boldsymbol{\mu}_{2}\right)$. If this is true under the induction assumption there exist an orthogonal matrix $\boldsymbol{U}_{N}$ (included in $\left.\boldsymbol{U}_{2}\right)$ such that the diagonal elements of $\operatorname{diag}\left(\boldsymbol{U}_{N}^{T} \boldsymbol{\Lambda}_{1} \boldsymbol{U}_{N}, \boldsymbol{U}_{N}^{T} \boldsymbol{\Lambda}_{2} \boldsymbol{U}_{N}\right)$ are precisely $x_{i 1}, x_{i 2}, \ldots, x_{i N}, x_{j 1}, x_{j 2}, \ldots, x_{j N}$.

We need to check conditions (22.a) and (22.b) again. For condition (22.a) it is enough to show that

$$
\begin{gathered}
\sum_{j=k}^{l} x_{i j} \leq \sum_{\substack{j=k+1 \\
l}}^{l} y_{i j}+\left(y_{i k}+y_{i N+1}-x_{i N+1}\right), \\
l=k, k+1, \ldots, 2 N-1
\end{gathered}
$$

By using (22.b) for the matrix of order $2 N$ we have

$$
\sum_{i=1}^{2 N-1} x_{i}=\sum_{i=1}^{2 N} x_{i}-x_{2 N}=\sum_{i=1}^{2 N} y_{i}-x_{2 N}=\sum_{i=1}^{2 N-1} x_{i}+y_{2 N}-x_{2 N}
$$


which can be written using (22.a) for the matrix of order $2 N+2$ as

$$
\sum_{i=1}^{j} x_{i} \leq \sum_{i=1}^{j} y_{i}+y_{2 N}-x_{2 N}, \quad j=1,2, \ldots, 2 N
$$

or equivalently

$$
\sum_{i=1}^{k-1} x_{i}+\sum_{i=k}^{j} x_{i} \leq \sum_{i=1}^{k-1} y_{i}+\sum_{i=k+1}^{j} y_{i}+\left(y_{k}+y_{2 N}-y_{2 N}\right)
$$

Since already $\sum_{i=1}^{k-1} x_{i} \leq \sum_{i=1}^{k-1} y_{i}$ the desired results follows. Condition (22.b) it easy to verify since

$$
\sum_{j=1}^{N+1} y_{i j}-x_{i N+1}+\sum_{k=1}^{N+1} y_{j k}-x_{j N+1}=\sum_{j=1}^{N} x_{i j}+\sum_{k=1}^{N} x_{j k}
$$

or

$$
\sum_{i=1}^{2 N+2} y_{i}=\sum_{j=1}^{N} x_{i j}+x_{i N+1}+\sum_{k=1}^{N} x_{j k}+x_{j N+1}
$$

which completes the proof of theorem for $N+1$.

iii) To verify the theorem for $M>2$ it is enough imposing in (23) a matrix $\boldsymbol{U}_{1}$ such that

$$
\boldsymbol{U}_{1}=\operatorname{diag}\left(\boldsymbol{I}_{N}, \boldsymbol{V}_{1}, \boldsymbol{I}_{N}, \boldsymbol{V}_{2}, \ldots, \boldsymbol{I}_{N}, \boldsymbol{V}_{M}\right)
$$

and

$$
\boldsymbol{U}_{2}=\operatorname{diag}(\underbrace{\left(\boldsymbol{U}_{N}, 1, \boldsymbol{U}_{N}, 1, \ldots, \boldsymbol{U}_{N}, 1\right)}_{M} .
$$

We need to follow (25)-(29) again considering a vector of length $M N$.

\section{ACKNOWLEDGMENT}

The author thanks Marcelo S. Alencar and Celia Desmond Editors-in-Chief, and the anonymous reviewers for helpful suggestions that improved the quality of this paper.

\section{REFERENCES}

[1] P. Cotae, M. Aguirre and M. Shadaram "Complex spreading sequence design for S-CDMA Systems under Total Weighted Squared Correlation”, 2006 IEEE Radio and Wireless Symposium 16-19 Jan.2006.

[2] P. Cotae, "Construction of complex-valued simplex signature sets with constant chip magnitude" IEEE Military Communications Conference, MILCOM 2003, 13-16 Oct. 2003, Vol. 2, pp.1196 - 1201, 2003.

[3] A. O. Boulakov and S.G Haggman, "System Aspects of Smart-Antenna technology in cellular wireless communications-an overview", IEEE Trans. on Microwave and Techniques. Vol. 48, No.6, pp. 919-929, Jun. 2000.
[4] G. N. Karystinos and D.A. Pados, "New bounds on the total squared correlation and optimum design of DS-CDMA binary signature sets", IEEE Trans. on Commun., Vol. 51, no.1, pp. 48-51, Jan 2003.

[5] G. N. Karystinos and D.A. Pados, "The maximum squared correlation, sum capacity, and total asymptotic efficiency of minimum total-squaredcorrelation binary signature sets”, IEEE Trans. Inform. Theory, Vol.51, pp 348-355, Jan 2005.

[6] P. Xia, S. Zhou, and G.B. Giannakis "Achieving the Welch bound with difference sets”, IEEE Trans. Inform Theory, vol.51, no.5, pp. 19001907, Jan. 2005.

[7] B.M. Hochwald, T.L. Marzeta, T.L. Richardson, W. Sweldens, and R. Urbanke, "Systematic design of unitary space-time constellations", IEEE Trans. Inform. Theory, vol. 46, no.6, pp 1962-1973, Sept. 2000.

[8] P. Cotae "Multicell spreading sequence design algorithm for overloaded S-CDMA", IEEE Communication Letters, vol.9, no.12, pp. 1028-1030, Dec. 2005.

[9] I. Oppermann and B.S. Vucetic, "Complex spreading sequences with a wide range of correlation properties”, IEEE Trans. on Commun. Vol. 45, no.3, pp. 365-375, March 1997.

[10] John C.L. Ng, K.B. Letaief and R.D. Murch, "Complex optimal sequences with constant magnitude for fast channel estimation initialization, IEEE Trans. on Commun. Vol. 46, no.3, pp. 305-308, March 1998.

[11] A. Milewski, "Periodic sequences with optimal properties for channel and fast start-up equalization”, IBM J. Res. Develop., Vol. 27, pp. 426431, Sept. 1983.

[12] W.H. Mow," A new unified construction of perfect root-of-unity sequences " in Proc. Int. Symp. Spread Spectrum Techniques and Its Applications, ISSSTA 1996, Mainz Germany, pp.955-959, 1996.

[13] H.H. Dam, H.J Zepernik and H. Luders, ”On the design of complex spreading sequences using a genetic algorithm" in Proc. Int. Symp. Spread Spectrum Techniques and Its Applications ISSSTA 2004, Sydney, Australia, 30 Aug-2 Sept., pp. 704 -707, 2004.

[14] B. Natarajan, S. Das, S. Stevens, " Design of optimal complex spreading codes for DS-CDMA using an evolutionary approach, IEEE Global Telecommunications Conference, GLOBECOM 2004, 29 Nov.-3 Dec., Vol.6, pp. 3882-3886, 2004.

[15] J.A. Tropp, I.S. Dhillon, R.W Heath, Jr., "Finite-step algorithms for constructing optimal CDMA signature sequences”, IEEE Trans. Inform. Theory, Vol. 50, no.11, pp.2916-2921, Nov.2004.

[16] P. Viswanath and V. Anantharam, "Optimal sequences and sum capacity of synchronous CDMA systems", IEEE Trans. Inform. Theory, Vol. 45, no.6, pp.1984-1983, Sept. 1999.

[17] N.N. Chan and K. -H. Li, "Diagonal elements and eigenvalues of a real symmetric matrix “, J. Math. Anal. Appl., Vol.91, pp 562-566, 1983.

[18] D. V. Sarwate, "Meeting the Welch bound with equality", Sequences and their Applications, pp. 79-102, 1998.

[19] L.R. Welch, "Lower bounds on the maximum cross correlation of signals” IEEE Trans. Inform. Theory, Vol.IT-20, pp397-399, May 1974.

[20] A.W. Marshall and I. Olkin, Inequalities: Theory of Majorization and Its Applications, New York: Academic, 1979.

[21] G.H. Golub and C.F. Van Loan, Matrix Computation, The John Hopkins University Press, 1996.

[22] R.A. Horn and C.A. Johnson, Matrix Analysis, Cambridge, U.K, Cambridge University Press, 1985.

[23] M.B. Pursley, "Direct sequence spread spectrum communications for multipath channels" IEEE Trans. on Microwave and Techniques, Vol. 50, no.3, pp. 653-661, March 2002.

[24] P. Cotae, M. Aguirre and M. Shadaram "An algorithm for unit tight frames with Constant Chip Magnitude Design in Overloaded CDMA Systems” Proceedings of CISS 2006, Annual Conference on Information Sciences and Systems, pp.1528-1533, Princeton, March 2006.

[25] P. Cotae "Transmitter adaptation algorithm for multicellular synchronous DS-CDMA systems with multipath”, IEEE Journal on Selected Areas in Communication, Vol. 24, no.1, pp. 94-103, Jan. 2006.

[26] P. Cotae "On the optimal sequences and total weighted square correlation of synchronous CDMA systems in multipath channels" Accepted to IEEE Transaction on Vehicular Technology, June 2006, to appear July 2007.

[27] P. Cotae, "On the Design of Real Multicellular DS-CDMA Spreading Sequences under Total Weighted Squared Correlation Criterion”, 
Proceedings 2004 IEEE Global Telecommunications Conference GLOBECOM 2004, Dallas, TX, USA, vol.6, pp. 3902-3906, Dec. 2004.

[28] P. Cotae, "Spreading Sequence Design for Multiple-Cell Synchronous DS-CDMA Systems under Total Weighted Squared Correlation Criterion", EURASIP Journal on Wireless Communications and Networking (Special Issue on Innovative Signal Transmission and Detection Techniques for Next Generation Cellular CDMA Systems) vol. 2004, no.1, pp. 4-11, Aug. 2004.

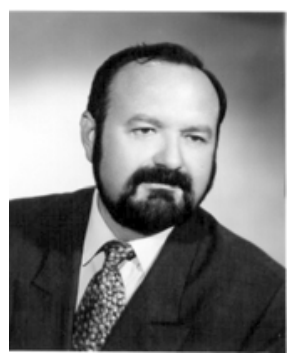

Paul Cotae (S'95-M'01-SM'04) was born on June 21, 1955 in Falticeni, Romania. He received a Dipl. Ing. and a M.S. degrees in communication and electronic engineering in 1980 from the Technical University of Iassy and a Ph.D. degree in telecommunications from Politechnica University of Bucharest, Romania in 1991. From 1984 to 2001, he was with the Department of Electrical Engineering, Technical University of Iassy, where he conducted research and teaching in the area of digital communications as a Full

Professor at the same department.

From 1994 to 1998 he spent four years at the University of Colorado at Colorado Springs and at the University of Colorado at Boulder as a Fulbright Scholar and Visiting Associate Professor doing research and teaching in Electrical and Computer Engineering department and Applied Mathematics department. He served also as a consultant to Navsys Corp., Colorado Springs, in 1997.

Since 2001, he has been with the University of Texas at San Antonio. His current research interests include multiple access, modulation and coding, mobile communications, and digital communication systems. He has authored or coauthored more than 100 papers in these areas and four books.

Dr. Cotae serves as an Associate Editor for IEEE Communication Letters, EURASIP Journal on Signal Processing, Elsevier International Journal of Computers and Electrical Engineering (JCEE), and he has been on the Technical Program Committee and Session Chair of the IEEE Conferences GLOBECOM (2003-2007), VTC Spring 2005, 2006, CISS 2006, WCNC 2006, SMC 2007, SoSE 2007, and ICC 2005-2008.

He is a Senior Member of IEEE, member of ASEE, member of HKN (Eta Kappa Nu) and SIAM. He is cited in Who's Who in American Education, Who's Who in America, and in Who's Who in the World. He has been nominated two times for the best teacher award (2005 and 2006) in the College of Engineering at UTSA. 